\title{
CDKN2A Protein Variant
}

National Cancer Institute

\section{Source}

National Cancer Institute. CDKN2A Protein Variant. NCI Thesaurus. Code C146926.

A variation in the amino acid sequence for a protein encoded by the CDKN2A gene. 